\title{
DEVICE FOR GEOPHYSICAL EXPLORATION OF THE EARTH'S CRUST BASED ON A DETONATION GENERATOR
}

\author{
Mamasadikov Yu . \\ Yunusaliev E.M. \\ Tojiev R.J.
}

Article DOI: https://doi.org/10.36713/epra2754

\begin{abstract}
ANNOTATION
The article describes the principle of operation of the device for geophysical exploration, which is based on the action of detonation waves to a limited area of the earth's surface at a shallow depth (up to $100 \mathrm{~m}$ ), a block diagram and timing diagrams explaining the principle of the device.

KEYWORDS: geophysical exploration, device, block diagram, principle of operation, timing diagram, detonation generator, phase shift, harmonic component of echo signals.
\end{abstract}

\section{INTRODUCTION}

The most important factors that increase the reliability and safety of the construction process in complex hydrogeological conditions, the accuracy and reliability source data when conducting survey works.

One of the most important parts of buildings and structures, is the Foundation, which is designed to transfer the load from all of the building structures on the base. But for the selection of constructive decisions of the Foundation must be clearly and know exactly what is the basis of what the engineering-geological elements it is represented, what mechanical and deformation properties [1]. The problem of finding different items, voids, and other inhomogeneities located beneath the surface of the earth, is very relevant during construction and archaeological work, as well as in many other spheres of economic activity [2].

In this regard, quite urgent is to develop a device for geophysical prospecting of the earth's crust, which provides sufficient performance registration information and the possibility of their repeated use.

The main part. For performance reasons, registration information and more reusability the authors developed a device for geophysical prospecting of the earth's crust on the basis of detonation generator block diagram is shown in Fig.1.

The device operates as follows. The synchronizing generator SG generates a sequence of short rectangular pulses with a repetition period (the maximum depth of exploration; - the speed of propagation of acoustic waves in the medium, Fig. 2, a time t1) which acts on the trigger input of the source of acoustic radiation of IAS, ИH1 first indicator and the second indicator $И \mathrm{H} 2$. In which the first and second $И \mathrm{H} 1 \mathrm{UH}_{2}$ indicator reset information and is prepared to receive regular information, at the same time the release of the first single-shot OV1 is formed a rectangular pulse (Fig. 2, b) which is supplied to the trigger input of the generator of short pulses GKI, solenoid gaseous hydrocarbon fuel ESC and solenoid valve compressed air EQ. At the same time, the open state of the solenoid valve EQ and ESC corresponds to the duration of the rectangular pulses generated by the first one-shot OV1, which is sufficient to completely fill uglevodorodnogo a mixture chamber of a detonation generator of DK.

With the trailing edge of the output pulse of the first single-shot OV1 (time t2 Fig. 2) is the generator of short pulses of GKI, the result at its output is formed a short square pulse, which simultaneously affect the input 
of the initiation system SI, the second one-shot OB2 and trigger with separate start SFS. Further, at the output of the latter is a logical "1" and is fed to the input of the meter time intervals EVIE.

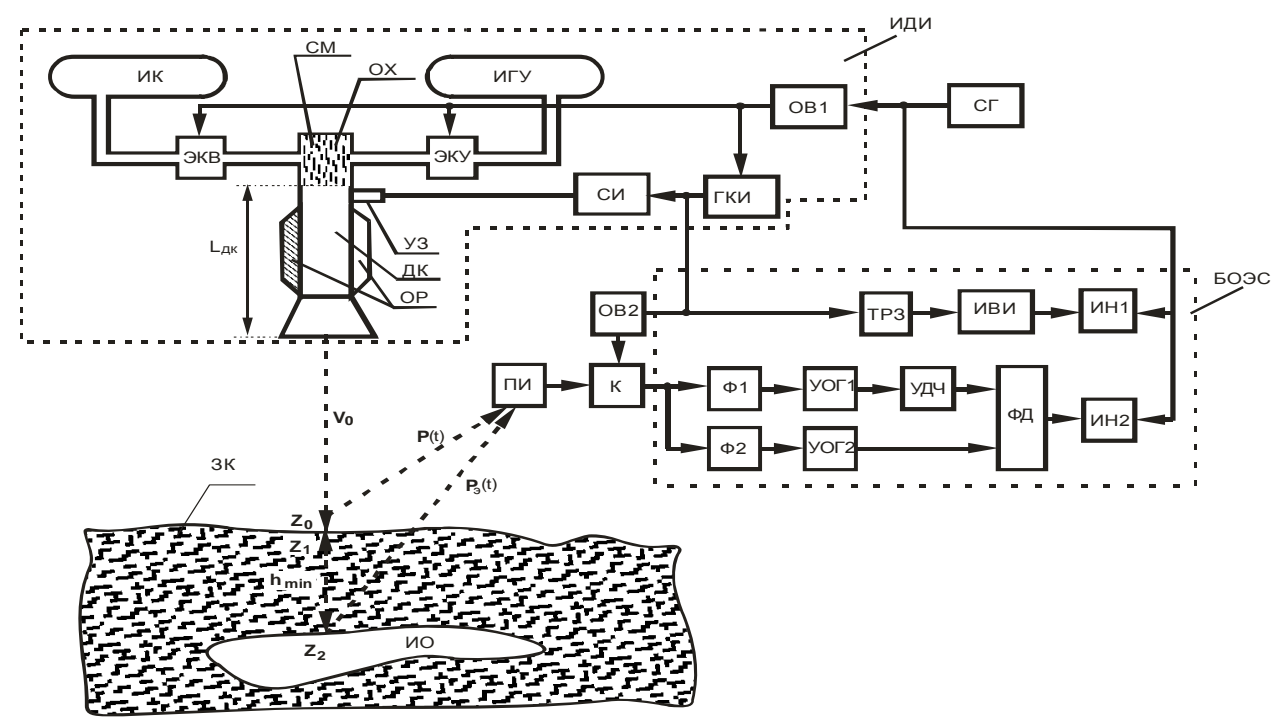

Fig. 1. Block diagram of a device for geophysical exploration of the earth's crust based on a detonation generator.

The impact of a short pulse from the output of the GKI short pulse generator to the input of the SI initiation system generates a high-voltage voltage pulse in its output (Fig. 2d). A high-voltage pulse from the output of the initiation system acts on the input of the ultrasonic ignition device and leads to ignition of the hydrocarbon-air mixture inside the detonation chamber. Further, due to the acceleration of the flame front inside the detonation chamber of a recreation center, a shock wave is formed at its output and acts on the earth's surface. At time t2, a rectangular pulse is generated at the output of the second one-shot OB2 (Fig. 2f), the duration of which is longer than the envelope of a multi-frequency damped oscillation, i.e., tOV2> t33K.

A rectangular pulse formed at the output of the second one-shot OB2 acts on the input of switch $\mathrm{K}$ and blocks the input of the BOES echo processing unit from the PI radiation receiver.

The impact of a shock wave on the earth's surface excites multi-frequency harmonic, damped seismic waves (Fig. 2, e), the waveforms and frequency composition of which depend on many factors, such as the nature of the impact pulse absorbing the properties of the medium, as well as structural features of the interface on the path waves, etc. [3].

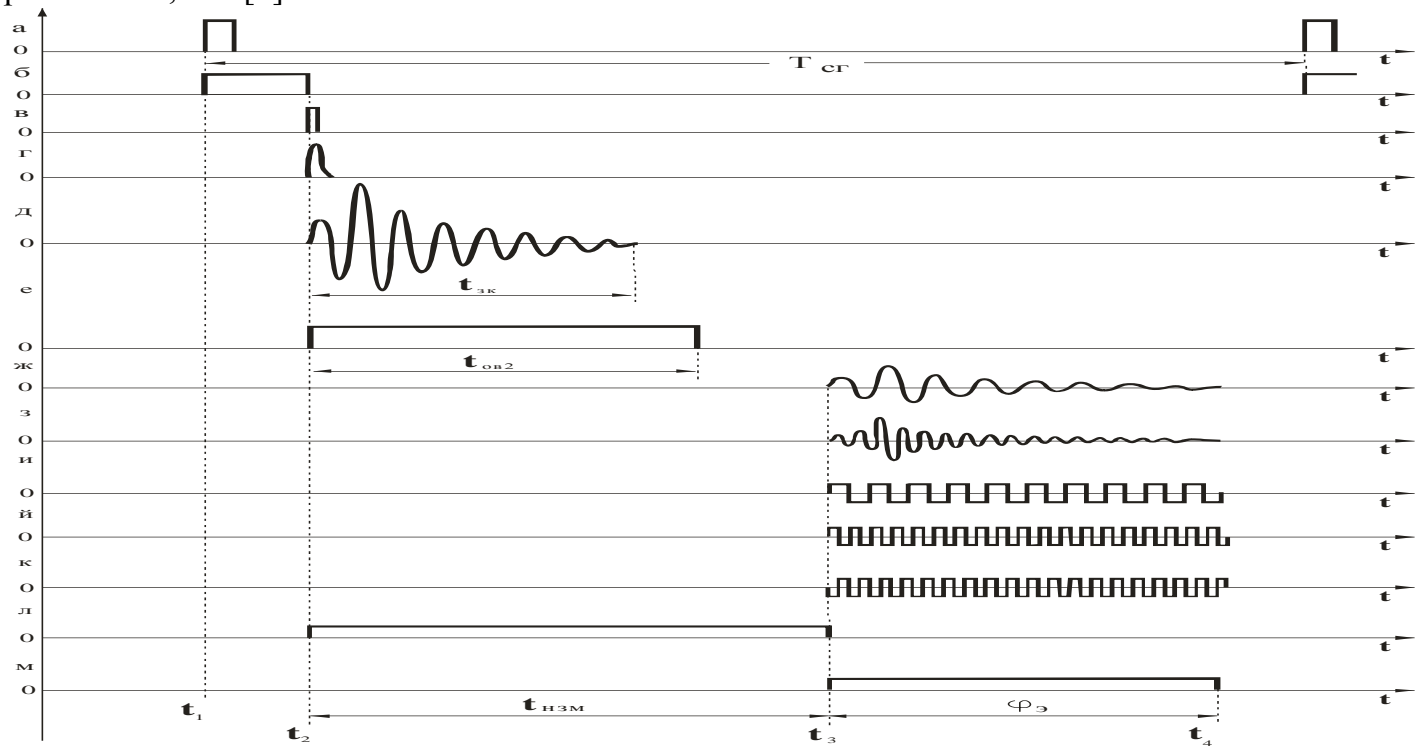

Fig. 2. Timing diagrams of the device.

In modern seismic exploration, powerful explosive sources are used, which have a pulse duration of several hundred milliseconds, and the frequency band of the spectrum of excited seismic waves is $1-200 \mathrm{~Hz}$ [4]. 
It is known that the frequency band of the spectrum of multifrequency, damped, seismic waves excited by a shock wave, with an increase in the pulse duration, narrows and vice versa with a decrease - expands, therefore when using detonation generators as an excitation source, the frequency band of excited seismic waves expands several times, so how the duration of the exposure pulse in detonation generators, depending on the length of the detonation tube, can range from several tens of microseconds to several milliseconds [5].

The required length of the detonation chamber is determined from the condition $L_{\partial \kappa} \leq \frac{V_{\partial}}{V_{3}} 2 h_{\text {min }}$, где: $h_{\text {min }}$ - minimum depth of the desired object; $V_{\partial} n V_{3}$ - the propagation velocity of the detonation wave and seismic waves at the depth of the prospected portion of the earth's crust, respectively [6-8].

According to $[9,10]$ and the foregoing, the formed harmonic, damped, seismic wave on the earth's surface

has the form: $P(t)=P_{1} \mathrm{e}^{-\alpha_{1} t} \sin \left(\omega_{1} t\right)+P_{2} e^{-\alpha_{2} t} \sin \left(\omega_{2} t\right)+\ldots+P_{n} e^{-\alpha_{n} t} \sin \left(\omega_{n} t\right)$

Where: $P_{1}, P_{2}, \ldots, P_{n}$ - maximum amplitude of harmonic components at frequencies

$\omega_{1}, \omega_{2}, \ldots, \omega_{\mathrm{n}}$ respectively; $\omega_{1}=2 \pi f_{1}, \omega_{2}=2 \pi f_{2}, \ldots, \omega_{n}=2 \pi f_{n}$ - circular frequency;

$\alpha_{1}, \alpha_{2}, \ldots, \alpha_{n}$ - media attenuation coefficients that correspond to frequencies $\omega_{1}, \omega_{2}, \ldots, \omega_{n}$ respectively; ttime.

From the theory of flaw detection, it is known that the main information about the nature of the desired object is contained in the phase shift of the echo signals $[9,11]$; therefore, the formed wave propagating along the earth's surface travels the distance hc and reaches the surface of the desired object, reflected from it, undergoing changes of the phase of each harmonic component, the distance hc passes once more and reaches the surface of the earth where the PI radiation receiver is installed (Fig. 2g), while the wave acting on the PI radiation receiver has the form. $P_{\ni}(t)=P_{\ni 1} e^{-\alpha_{1} t} \sin \left[\omega_{1}\left(t-\tau_{3}\right)+\varphi_{1}\right]+P_{\ni 2} e^{-\alpha_{2} t} \sin \left[\omega_{2}\left(t-\tau_{3}\right)+\varphi_{2}\right]+\ldots$ $\ldots+P_{\ni n} e^{-\alpha_{n} t} \sin \left[\omega_{n}\left(t-\tau_{3}\right)+\varphi_{n}\right]$

Where: $P_{\ni 1}, P_{\ni 2}, \ldots, P_{\ni n}$ - maximum amplitude of harmonic components of echo signals at frequencies $\omega_{1}, \omega_{2}, \ldots, \omega_{n}$ respectively;

$\varphi_{1}, \varphi_{2}, \ldots, \varphi_{n}$ - frequency shift of echoes at frequencies $\omega_{1}, \omega_{2}, \ldots, \omega_{n}$ respectively; - delay $\tau_{3}$ time of echoes corresponding to the distance $-2 h_{\mathrm{L}}$.

The electrical signal generated at the output of the PI radiation receiver has the form.

$$
\begin{aligned}
& \mathrm{U}_{\ni}(t)=U_{\ni 1} e^{-\alpha_{1} t} \sin \left[\omega_{1}\left(t-\tau_{3}\right)+\varphi_{1}\right]+U_{\ni 2} e^{-\alpha_{2} t} \sin \left[\omega_{2}\left(t-\tau_{3}\right)+\varphi_{2}\right]+\ldots \\
& \ldots+U_{\ni n} e^{-\alpha_{n} t} \sin \left[\omega_{n}\left(t-\tau_{3}\right)+\varphi_{n}\right]
\end{aligned}
$$

Where: $U_{\ni 1}, U_{\ni 2}, \ldots, U_{\ni n}$ - maximum voltage amplitude of the harmonic components of the echo signals at frequencies $\omega_{1}, \omega_{2}, \ldots, \omega_{n}$ respectively.

According to the theory of acoustic control methods, to assess the nature of the desired object, it suffices to measure the phase shift between two adjacent harmonic components of the echo signals, which differ in frequency $[9,11]$.

The choice of the corresponding pair of harmonic components of the echo signals is made according to their more intense amplitude, which in seismic exploration using powerful explosive sources corresponds to the frequency range of $20-80 \mathrm{~Hz}[3,4]$.

It has been experimentally established that when using detonation generators, which have a pulse duration in the order of one millisecond, the frequency range at levels of 0.5 of the maximum amplitude of the echo signals is $20-400 \mathrm{~Hz}$, which allows the use of more high-frequency, harmonic, components of the echo signals for determination and classification the desired object at a depth of $100 \mathrm{~m}$.

It is known that in phase metering to measure phase shifts between a component of harmonic signals, it is necessary to bring them to the same frequency by multiplying their initial frequency. 
Then the phase shift between the selected harmonic components at a frequency of their frequency according to is defined as

$$
\omega_{m}=k \omega_{0}, \omega_{(m+1)}=(k+1) \omega_{0}
$$

Bringing them to the same frequency, for example $\omega=k(k+1) \omega_{0}$, by multiplying frequencies $\omega_{1}$ and $\omega_{(m+1)}$ respectively on $(k+1)$ and $k$, allows you to determine the phase difference between the harmonic components of the echo signals.

$$
\psi=(k+1) \varphi_{m}-k \varphi_{(m+1)}
$$

Where: $m=1,2, \ldots$ - number of the selected harmonic component.

When the frequencies of the selected harmonic components $\omega_{m}$ and $\omega_{(m+1)}$ slightly differ from each other, then you can take $\varphi_{m}=\varphi_{(m+1)}=\varphi_{\ni}$.

Therefore, the phase difference between the harmonic components will be equal to the phase shift of the echo signal $\psi=\varphi_{\ni}$ (Fig. $2 \mathrm{~m}$ ).

The implementation of this method of measuring the phase shift between the harmonic components of the echo signals is performed in the block processing of the echo signals of BOES (Fig.1).

This electric output signal of the radiation receiver PI is applied through the switch To the input of the processing unit of the echo of BOES.

The electrical signal corresponding to the reflected acoustic signal from the surface of the earth $\mathrm{P}(\mathrm{t})$ is blocked by switch To period of time of a damped oscillatory process on the earth's surface (Fig. 2. W), and the electric signal corresponding to the reflected echo from the surface of the object re(t) passes through the switch after a time interval t OB2 switch is in the locked state by the output pulse of the second one-shot S (Fig. 2. e).

Passing through the switch an electrical signal which corresponds to the reflected echo from the surface of the desired object IO, are simultaneously energized on the input of the first F1 and second F2 filter. The electric signal (Fig. 2) corresponding to the frequency output from the first filter F1 passes through the amplifier limiter УОГ1 (Fig. And 2) is then fed to the second input of the trigger with separate run SFS and through the frequency doubler ADC to one of the inputs of the phase detector FD (Fig. 2. th). To the second input of the phase detector PD is fed a corresponding electrical signal (Fig. 2) with a frequency which is received through the second amplifier limiter УОГ2 output from the second filter F2.

Thus, the output of the phase detector PD is formed an electric signal proportional to the phase shift between harmonic components of the echo (Fig. $2 \mathrm{~m}$ ), and the output of the trigger with separate run, is formed a rectangular pulse whose duration corresponds to the distance to the desired object $\mathrm{z}$, and is measured on the meter of time intervals EVIE. Signal corresponding to the distance $\mathrm{z}$ is displayed on the display $\mathrm{UH} 1$, and the display $И \mathrm{H} 2$ shows a signal corresponding to a phase shift $\psi=\varphi_{\ni}$

Conclusion The use of the proposed device in geophysical exploration based on detonation generators in the construction industry makes it possible to determine and classify underground formations, objects, voids and other inhomogeneities at a depth of up to $100 \mathrm{~m}$, which is due to the use of higher-frequency harmonic components of echo signals in the range of $20-400 \mathrm{~Hz}$. Improving the accuracy of measurements is achieved by eliminating the influence of reflected signals from the earth's surface on the control result.

\section{REFERENSES}

1. A. J. Rump, D. P. Dasaev. The prospect of the development of automated systems for testing of construction properties of soils// prospects of development of construction complex. 2013. Volume1. P. 126-130.

2. Mambetov A. sh., sh. Mambetov A. Geoacoustic methods of studying rock mass. Bishkek: Izd-vo KRSU, 2018. 130 C.

3. Gurvich I. I., Bohanec GN Seismic exploration: a Textbook for vuzov.-3-e Izd., Rev. M.: Nedra, 1980, p. 551

4. Znamenskiy V. V., Zhdanov, M. S., Petrov L. P. Geophysical methods of prospecting and exploration of wells. M.: Nedra, 1981, 320 p.

5. Tojiev. R. J. the Mechanical-technological solutions contactless influence on soil and plant development gazobetonnyh units for high-efficiency of cotton cultivation: dis. ...Dr. of technical Sciences: 05.20.01/Tojiev Rasuljon Jumabaevich. - M.: 1993. - 358.

6. Mamasadykov Yoo, Defended E. M. etc. a Device for excitation of acoustic waves // Modern problems of design, construction and operation of engineering communication systems: proceedings of the respub. Conf. TACI Tashkent -2017. part-II, p. 218-220. 
7. R. J. Tojiev, Masadykov Yu etc. an Acoustic method for the modern exploration of the earth's crust. "Ecologic toza Isla Halik maculature or Ales muammolari" Repub. Ilmi-Amali Conf. materiallari. (Parona, 15-16 may 2007) Parana. 2007. - 35-37-b.

8. Masadykov Mamasolieva Z. Y. Yu and etc. the Acoustic method for geophysical exploration of the shallow depth of the crust. "Islam cialisno modernizational, appliances VA tekhnologik it islahd of innovasiyalar, itiso-DIY samarador of usullar VA noanyone ecim-lar" resp.Elmi-tech. Anjum. Mayr.thesis. (Farone, 30-31мaŭ 2008). Farona.2008. - $73-75-b$.

9. Mamasadykov Yu. M., Defended E. M. Device for geophysical exploration // scientific-technical journal + Ferpi, 2017, no. 4, pp. 223-226.

10. Y. M. Mamasadikov, E. M. Defended. Acoustic method for determination and classification of underground structures and the structure of the earth's crust in the construction of industrial facilities. "Muhendislik communication of isimlerini loyal, URIs VA modernizacijskim zamonaviy of masalalari" mavzusida halara of Ilmitech conference materiallari. Samrand 2014. (20-21 may) 221-223 b.

11. Rzhevkin S. N. A course of lectures on the theory of sound. M., 1960. c. 337.

12. Sagdiyev, H. S., \& Defended The, E. M. (2010). Vibrations of soil and structures in the industrial explosions in complex geological conditions. Tashkent: FAN. 